\title{
Retraction Note: Predicting adult height from DNA variants in a European-Asian admixed population
}

\author{
Xiaoxi Jing ${ }^{1} \cdot$ Yanan Sun ${ }^{2,3} \cdot$ Wenting Zhao ${ }^{2} \cdot$ Xingjian Gao ${ }^{1} \cdot \mathrm{Mi} \mathrm{Ma}^{2,4} \cdot$ Fan Liu $^{1,5} \cdot$ Caixia Li $^{2}$ \\ Published online: 7 September 2021 \\ ๑) Springer-Verlag GmbH Germany, part of Springer Nature 2021
}

\section{Retraction Note to: International Journal of Legal Medicine (2019) 133:1667-1679 https://doi.org/10.1007/s00414-019-02039-8}

The Editors-in-Chief have retracted this article. Since publication, concerns were raised about the ethics and consent procedures for this study. We requested supporting documentation from the authors, including the application form submitted to the ethics committee and evidence of ethics approval. The documents supplied by the authors contain insufficient information related to the scope of the study for us to remain confident that the protocols complied with our editorial policies or are in line with international ethical standards [1].

Caixia Li stated on behalf of all co-authors that they do not agree to this retraction.

The original article can be found online at https://doi.org/10.1007/ s00414-019-02039-8

Fan Liu

liufan@big.ac.cn

$\triangle$ Caixia Li

licaixia@tsinghua.org.cn

1 CAS Key Laboratory of Genomic and Precision Medicine, Beijing Institute of Genomics, University of Chinese Academy of Sciences, Chinese Academy of Sciences, Beijing, China

2 National Engineering Laboratory for Forensic Science, Key Laboratory of Forensic Genetics of Ministry of Public Security, Beijing Engineering Research Center of Crime Scene Evidence Examination, Institute of Forensic Science, Ministry of Public Security, Beijing, China

3 Institute of Forensic Medicine and Laboratory Medicine, Jining Medical University, Jining, China

4 Xinjiang Production and Construction Corps of Seventh Division Public Security Bureau, Ürümqi, China

5 Department of Genetic Identification, Erasmus MC University Medical Center Rotterdam, Rotterdam, The Netherlands

\section{Reference}

1. World Medical Association (2001) World Medical Association Declaration of Helsinki. Ethical principles for medical research involving human subjects. Bull World Health Organ 79(4):373

Publisher's note Springer Nature remains neutral with regard to jurisdictional claims in published maps and institutional affiliations. 